medgen $2018 \cdot 30: 389-390$

https://doi.org/10.1007/s11825-018-0220-4

Online publiziert: 28. November 2018

(c) Springer Medizin Verlag $\mathrm{GmbH}$, ein Teil von Springer Nature 2018

CrossMark
Bodo Beck - Christian Netzer

Institut für Humangenetik der Uniklinik Köln, Köln, Deutschland

\title{
Einführung zum Thema: Erbliche Nierenerkrankungen
}

\section{Die Nephrogenetik wird erwachsen}

Nach 18 (sic!) Jahren erscheint wieder ein Themenheft zu erblichen Nierenerkrankungen in der Zeitschrift „medizinischegenetik“. Die Auswahl der Krankheitsbilder haben wir aus dem im Jahr 2000 von Klaus Zerres koordinierten Heft zu großen Teilen übernommen - ebenso wie den Titel. Beides möchten wir als Kompliment an die damaligen Autoren verstanden wissen. Und doch zeigt das Blättern in der alten Ausgabe, welche Zeitenwende die Humangenetik mittlerweile durchlebt hat. Die damaligen Tabellen enthielten in der Spalte „Ursächliche Gene“ oft nur einen oder wenige Einträge und zudem viele Fragezeichen. Zum Teil konnte nur ein chromosomaler Locus angegeben werden. Die Übersichtsarbeiten des vorliegenden Heftes hingegen quellen förmlich über vor krankheitsassoziierten Genen.

Eine direkte Folge der Genomik-Ära ist, dass das vertiefte molekulare Verständnis vieler Krankheitsbilder in Kombination mit neuen Technologien (wie RNA-Interferenz-, CRISPR/CAS9-, und/ oder Stammzell-basierte Ansätzen) nun zahlreiche Früchte trägt: Während im alten Heft therapeutische Aspekte - wenn überhaupt - meist nur kurz angesprochen werden konnten, enthält beispielsweise der neue Beitrag über das AlportSyndrom eine eigene Tabelle zu aktuellen klinischen Studien in Deutschland und ein Flussdiagramm zur medikamentösen Therapie. Hier werden erste Konturen der vielzitierten „Präzisionsmedizin“ erkennbar.

Der Stellenwert der genetischen Diagnostik ist für die Nephrologie des Jahres 2018 sicherlich auch deutlich höher als noch zu Beginn des neuen Jahrtau- sends. Trotz der ungleich größeren Zahl an relevanten Genen, die es $\mathrm{zu}$ testen gilt, sind die Sequenzierkosten und -zeiten dank Next-Generation Sequencing erheblich gesunken. Auch hinsichtlich ihres patientenorientierten Nutzens ist die molekulargenetische Diagnostik also gewissermaßen erwachsen geworden. Dies dürfte einer der Gründe sein, warum die genetische Abklärung heute in vielen Fällen am Anfang und nicht mehr am Ende des diagnostischen Algorithmus steht. Ein anderer Grund ist die bereits angesprochene therapeutische Relevanz einer exakten molekularen Diagnose. Durch entsprechende Gesetzesänderungen in den USA und in der EU werden zunehmend Medikamente für Seltene Erkrankungen entwickelt und zugelassen, auch auf dem Gebiet der Nephrologie. Diese therapeutischen Innovationen sind allerdings oft teuer. So belaufen sich die Jahrestherapiekosten von Eculizimab (Soliris ${ }^{\circledR}$ ) zur - sehr effektiven Behandlung des atypischen hämolytischurämischen Syndroms auf mehrere hunderttausend Euro. Naheliegender Weise wird mit Hilfe einer frühzeitigen genetischen Diagnostik versucht, eine solche Therapie möglichst nur denjenigen $\mathrm{Pa}$ tienten zukommen zu lassen, bei denen sie auch wirken kann.

Die Ausweitung der genetischen Diagnostik bringt aber auch alte Gewissheiten ins Wanken und zeigt, dass die Unterscheidung zwischen nephrologischen Krankheitsbildern in der Pädiatrie und der Erwachsenenmedizin oft nur in unseren Köpfen existiert. So konnte kürzlich bei einem überraschend hohen Anteil $(0,5 \%$ !) nierentransplantierter Erwachsener mit spätmanifester Niereninsuffi- zienz und der prototypischen Diagnose eines „chronischen Nierenversagens unklarer Ätiologie“ eine homozygote Deletion des NPHP1-Gens nachgewiesen werden - und damit eine der häufigsten Ursachen der Nephronophthise und der terminalen Niereninsuffizienz des Kindesalters. Die noch immer verbreitete Lehrmeinung, dass monogene Ursachen einer schweren Nierenfunktionsstörung - von der autosomal-dominant erblichen polyzystischen Nierenerkrankung einmal abgesehen - im Wesentlichen bei pädiatrischen Patienten zu finden sind, ist angesichts der Tatsache, dass in dieser Studie nur auf eine einzige pathogene Mutation aus der genetisch sehr heterogenen Krankheitsgruppe der Nephronophthisen getestet wurde, sicher revisionsbedürftig.

Wie in anderen Gebieten der Humangenetik beginnen auch in der Nephrogenetik in Folge der immer umfassenderen genetischen Diagnostik die Grenzen zwischen ehemals in den Lehrbüchern klar getrennten Krankheitsbildern bisweilen zu verschwimmen. So zeigt sich zum Beispiel, dass eine fokal-segmentale Glomerulosklerose (FSGS) mit klinischen und histopathologischen Mitteln alleine nicht immer klar von einem Alport-Syndrom zu unterscheiden ist. Eine eindeutige Diagnose - mit enormen Auswirkungen für die weitere Therapie und die Einschätzung des Wiederholungsrisikos für $\mathrm{Fa}$ milienangehörige - ist in diesem Fällen erst durch den Nachweis der ursächlichen Mutation(en) möglich.

Eine umfassende nephrogenetische Diagnostik sollte auch zunehmend in der Pränatalmedizin an Bedeutung gewinnen. Beispielhaft sei hier auf zystische 
Nierenveränderungen des Feten hingewiesen, die eine Vielzahl von genetischen Ursachen haben können. Einige der zugehörigen Krankheitsbilder haben deutlich andere prognostische Implikationen als die autosomal-rezessiv erbliche polyzystische Nierenerkrankung (ARPKD), die heute noch oft reflexhaft aufgrund des Ultraschall-Befundes vermutet wird. Die Vorteile einer molekulargenetisch gesicherten Diagnose für das weitere Management der Schwangerschaft und für die Beratung der Schwangeren liegen auf der Hand.

Die nephrogenetische Forschung ist noch weit davon entfernt, alle monogen erblichen Formen von Nierenerkrankungen und -fehlbildungen aufgeklärt zu haben. Vieles gilt es noch zu entdecken und zu verstehen, beispielsweise auf dem Gebiet der Congenital Anomalies of the Kidney and Urinary Tract (CAKUT). Hier beträgt die diagnostische Aufklärungsrate umfassender genetischer Tests erst maximal $40 \%$. Wir hoffen, mit dem vorliegenden Themenheft einen Überblick über den Stand der Grundlagenforschung und der Translation des Wissens in die klinische Anwendung auf dem faszinierenden Forschungsgebiet der $\mathrm{Ne}$ phrogenetikzu liefern. Wir bedanken uns herzlich bei den Autoren der Beiträge und sind jetzt schon gespannt darauf, wie das Themenheft „Erbliche Nierenerkrankungen 3.0“ im Jahr 2036 aussehen wird!

\section{Korrespondenzadresse}

Dr. med. Bodo Beck

Institut für Humangenetik der Uniklinik Köln Kerpener Str. 34, 50931 Köln, Deutschland bodo.beck@uk-koeln.de

Interessenkonflikt. B. Beck und C. Netzer geben an, dass kein Interessenkonflikt besteht.

\section{Sind wir immun gegen die Genschere CRISPR-Cas9?}

Die Genschere CRISPR-Cas9 weckt in der Gentherapie große Hoffnungen, Erbkrankheiten auf molekularbiologischer Ebene behandeln zu können. Wissenschaftlerinnen und Wissenschaftler der Charité - Universitätsmedizin Berlin haben jetzt Reaktionen des menschlichen Immunsystems auf CRISPR-Cas9 untersucht. Sie haben festgesellt, dass bei Menschen eine breite Immunität gegen das Protein Cas9 besteht. Um verschiedene Anwendungen der Genschere sicher zu machen, arbeiten die Forscher an ersten Lösungsansätzen. Über die Chancen und Risiken von CRISPR-Cas9 berichten sie in Nature Medicine.

Mit CRISPR-Cas9 lässt sich eine DNA-Sequenz zerschneiden, ausschalten oder durch eine andere ersetzen - entsprechend wird es auch als Genschere bezeichnet. Das Verfahren kommt in zwei unterschiedlichen Varianten zum Einsatz: Entweder werden Zellen außerhalb des Körpers verändert oder erkrankte Körperzellen werden direkt im Organismus modifiziert. In beiden Fällen zeigt die Nutzung im Tiermodell bislang eine einfache und effiziente Handhabung.

Gleichwohl ist die Methode noch nicht hinreichend erforscht, um eine fundierte RisikoNutzen-Bewertung zur Anwendung beim Menschen vornehmen zu können. Diese Lücke hat das Team um Dr. Michael SchmückHenneresse vom Berlin-Brandenburger Centrum für Regenerative Therapien (BCRT) der Charité nun zu einem Teil geschlossen, indem sie die Immunreaktionen auf das CRISPR-Cas9-System erforscht haben.

\section{Körpereigene T-Zellen gegen Cas}

„Ein wesentlicher Bestandteil von CRISPRCas9 ist das Eiweißmolekül Cas9, das aus Streptokokken stammt. Da sich Menschen häufig mit diesen Bakterien infizieren, vermuteten wir, dass bereits ein immunologisches Gedächtnis gegen Cas9 bestehen könnte", erklärt Dr. Schmück-Henneresse. Bei fast allen gesunden Erwachsenen konnten die Wissenschaftler körpereigene Immunzellen, sogenannte T-Zellen, nachweisen, die auf Cas-Eiweißmoleküle reagieren. Auch CasMoleküle aus anderen Bakterienstämmen, wie Staphylokokken und Darmbakterien, bewirken solche Immunreaktionen, was an der hohen Ähnlichkeit der Enzyme liegen könnte. „Solche Immunzellen könnten zu unerwünschten Immunreaktionen bei der Gentherapie führen, was ihre Sicherheit und Effektivität beeinträchtigen könnte. Diese Gefahr besteht eventuell auch bei CRISPRCas9, darauf müssen wir uns vorbereiten", sagt der Studien-Erstautor Dimitrios
Laurin Wagner, Doktorand am BCRT und Promotionsstipendiat des Berlin Institute of Health (BIH).

\section{Immunreaktionstest entwickelt}

Für die Veränderung von Zellen außerhalb des Körpers bedeutet dies, dass Patienten keine Zellen injiziert werden sollten, während CRISPR-Cas9 in innen noch aktiv ist. „Um sicherzustellen, dass Zellen bedenkenlos angewendet werden können, haben wir einen Test entwickelt. Damit kann zuverlässig nachgewiesen werden, dass das Risiko einer Immunreaktion gering ist", erläutert Dr. Schmück-Henneresse. Manche genetischen Erkrankungen führen jedoch zu Fehlern in Geweben, die sich nicht außerhalb des Körpers verändern lassen. Hierfür müssen neue Lösungen gefunden werden, um gefährliche Immunreaktionen gegen die Genschere zu verhindern

Um Krankheiten sicher mit CRISPRCas9 behandeln zu können, haben die Wissenschaftler in Zusammenarbeit mit dem Berlin Center for Advanced Therapies (BeCAT) der Charité eine Technologie entwickelt. Diese soll regulatorische T-Zellen, die die unerwünschten Immunreaktionen kontrollieren, isolieren und vermehren. Erste klinische Versuche, bei denen dieser Ansatz bei Patienten nach einer Nierenoder Knochenmarktransplantation genutzt wurde, zeigen positive Ergebnisse.

Wagner DL et al. High prevalence of Streptococcus pyogenes Cas9-reactive T cells within the adult human population. Nature Medicine. 2018 Oct 29. doi: 10.1038/s41591-0180204-6.

\section{Manuela Zingl, Charité - Universitätsmedizin Berlin}

